\title{
The Contribution of Visual Sensitivity, Phonological Processing, and Nonverbal IQ to Children's Reading
}

\author{
Kristen Pammer and Alison Kevan \\ The School of Psychology \\ The Australian National University
}

\begin{abstract}
It has been suggested that the differences observed for dyslexic readers compared to normal readers on tasks measuring visual sensitivity may simply be the result of differences between the two groups in general cognitive ability and/or attentional engagement. One common way to accommodate this proposal is to match normal and dyslexic readers on IQ. However, an explicit test of this suggestion is to take normal and dyslexic readers who differ on IQ- - where IQ would be expected to explain reading ability—and determine if visual sensitivity can still account for reading skill, even when IQ is taken into account. In this study we explored the relative contributions of nonverbal IQ, visual sensitivity as measured by sensitivity to the frequency doubling illusion, and phonological and irregular word reading to reading ability. Visual sensitivity explained a significant amount of variance in reading ability, over and above nonverbal IQ, accounting for $6 \%$ of the unique variance in reading ability. Moreover, visual sensitivity was related primarily to irregular word reading rather than to nonsense word decoding. This study demonstrates that low-level visual sensitivity plays an intrinsic role in reading aptitude, even when IQ differences between normal and dyslexic readers are contrived to maximize the contribution of IQ to reading skill. These results challenge the suggestion that impaired visual sensitivity may be epiphenomenal to poor reading skills.
\end{abstract}

Reading is a complex skill that requires the successful translation of visual symbols into meaning, and learning to read requires explicit instruction in the visual characteristic of words, while making the subsequent link to language (Stein, 2003; Stein \& Walsh, 1997). Most children acquire this skill with little difficulty;

Correspondence should be sent to Kristen Pammer, School of Psychology, Faculty of Science, Canberra, Australia 0200. E-mail: kristen.pammer@anu.edu.au 
however, for approximately $10 \%$ of children, learning to read proves to be extremely difficult (Shaywitz, 1998). These children suffer from developmental dyslexia (hereinafter referred to as dyslexia), a specific learning disability in which an individual demonstrates an unexpectedly low reading ability despite normal intelligence, sensory acuity, motivation, social adjustment, and educational opportunity (Snowling, 2001; Stein \& Walsh, 1997; Vellutino, Fletcher, Snowling, \& Scanlon, 2004). The most prominent and widely acknowledged impairment in dyslexic readers is a difficulty in phonological processing, which typically accounts for large amounts of variance in reading skill, even after age and intelligence have been controlled (Francks, MacPhie, \& Maonaco, 2002; McDougall, Hulme, Ellis, \& Monk, 1994; Snowling, 2001).

However, considerable evidence has accumulated to suggest that in addition to their poor phonological skills, a proportion of dyslexic readers also have a visual processing deficit: Dyslexic readers respond differently to normal readers on visual tasks that require processing by the dorsal visual pathway, which is primarily driven by magnocellular input (e.g., Buchholz \& McKone, 2004; Casco, 1993; Cornelissen \& Hansen, 1998; Cornelissen et al., 1998; Demb, Boynton, Best, \& Heeger, 1998; Eden et al., 1996; Galaburda \& Livingstone, 1993; Hayduk, Bruck, \& Cavanagh, 1996; Keen \& Lovegrove, 2000; Kinsey, Rose, Hansen, Richardson, \& Stein, 2004; Lovegrove et al., 1982; Pammer \& Wheatley, 2001; Rayner, Pollatsek, \& Bilsky, 1995; Vidyasagar \& Pammer, 1999). These data have been adduced as supporting what has become known as the magnocellular deficit theory of dyslexia.

Nevertheless, the evidence of a magnocellular deficit in dyslexia has been questioned on several grounds. Some studies have failed to demonstrate visual processing differences between dyslexic and normal readers on tasks believed to be dependent on magnocellular functioning (e.g., Amitay, Ben-Yehudah, Banai, \& Ahissar, 2002; Ben-Yehuda, Sackett, Malchi-Ginzberg, \& Ahissar, 2001; Olson \& Datta, 2002; Ramus et al., 2003; Stuart, McAnally, \& Castles, 2001). These conflicting findings may be due to the use of reading-disabled children with different cognitive profiles (Hogben, 1996) or to the possibility that the magnocellular deficit theory needs to be further refined to describe the exact nature of the visual deficit. Moreover, researchers have highlighted the shared cognitive requirements between reading and psychophysical tests of dorsal system activity, suggesting that the standard measurements of both skills requires high levels of sustained attention and general cognitive ability (e.g., Stuart et al., 2001). A number of studies have confirmed the link between reading, IQ, and sensory skill. For example, Hulslander et al. (2004) failed to demonstrate a relationship between visual sensitivity and reading ability after statistically controlling for IQ. Similarly, much of the relationship between visual sensitivity and reading found by Olson and Datta (2002) was also explained by IQ. However, Talcott et al. (2002) and Talcott et al. 
(2000) demonstrated that visual sensitivity significantly predicted literacy skills, even after nonverbal IQ and age were taken into account.

Visual tasks such as coherent motion (Talcott et al., 2002; Talcott et al., 2000) and contrast sensitivity (Olson \& Datta, 2002) have been used to determine the relationship between cognitive attainment and reading ability. Although such tasks have been successful in discriminating between dyslexic and normal readers and in predicting reading ability, others have challenged these findings (see Amitay et al., 2002; Skottun, 2000; Skoyles \& Skottun, 2004; Stuart et al., 2001). The rationale for the use of visual measures such as coherent motion is the neurophysiological evidence that motion sensitivity is mediated by the dorsal visual pathway. This is a perfectly rational approach if one is trying to reveal a processing deficit in the dorsal pathway. However, motion discrimination is a reasonably complex skill: V5/MT, the part of the brain primarily responsible for motion discrimination, has a role in the complex integration of visual stimuli from multiple spatial sources (Vanni et al., 2004). As such, it is not surprising that visual tasks such as coherent motion, reading, and IQ share a large amount of common variance given the shared cognitive load in the neurobiological reading network, and it also may go some way toward explaining the variability demonstrated in the literature regarding the success or failure of tasks such as coherent motion and contrast sensitivity in predicting reading ability.

In this study we attempt to reduce the contribution of higher level cognitive processes to performance on visual sensitivity tasks by using sensitivity to the frequency doubling illusion (FD) as our measure of magnocellular/dorsal stream processing. The FD illusion is believed to be processed at the retinal level of the retinocortical visual pathway; the FD illusion, originally described by Kelly (1966), consists of sinusoidal grating patterns between 0.1 and 4 cycles/deg, which, when counterphase flickered at rates above $15 \mathrm{~Hz}$, create an illusory grating that is double the actual spatial frequency (Kelly, 1966, 1981; Tyler, 1974). As the FD effect is seen under monocular as well as binocular viewing conditions, it is suggested to be retinal in origin, produced by $\mathrm{M}(\mathrm{y})$ cells, a specific subgroup of magnocellular retinal ganglion cells (Bedford, Maddess, Rose, \& James, 1997; Maddess et al., 1999; Tyler, 1974). M(y) cells are the largest of all the retinal ganglion cells and are the largest cells in the magnocellular pathway, ranging in size from 50 to $150 \mathrm{~mm}$ in diameter (Benardete, Kaplan, \& Knight, 1992; Maddess \& Henry, 1992). Accordingly, M(y) cells have large receptor fields that respond over a large area of visual space (Kelly, 1981; Maddess et al., 1999). M(y) cells also have the fastest conduction velocities among the various retinal ganglion cells and demonstrate nonlinear activity (Benardete et al., 1992). Therefore the doubling seen in the FD illusion is proposed to be the result of the second harmonic distortion in the $\mathrm{M}(\mathrm{y})$ cells as the remainder of the retinal ganglion cells are linear and have little to no second harmonic distortion (Maddess \& Henry, 1992). As a result, 
this unique response of $\mathrm{M}(\mathrm{y})$ cells to the FD illusion makes the detection and measurement of the activity of these cells relatively easy.

A number of studies have now demonstrated that dyslexic readers are less sensitive to seeing the FD illusion than normal readers (Buchholtz \& McKone, 2004; Pammer, Lavis, \& Cornelissen, 2004; Pammer \& Wheatley, 2001). Because the FD illusion is mediated by $\mathrm{M}(\mathrm{y})$ cells, it is most likely to measure the integrity of magno cells (and therefore the dorsal pathway) at the level of the retina and is therefore likely to be less influenced by "higher order" cognitive skills. Hence it may be a more appropriate task to measure the relationship between visual sensitivity, reading, and IQ. What might an $\mathrm{M}(\mathrm{y})$ cell deficit tell us about reading difficulties? If a deficit in the dorsal stream contributes to reading difficulties, then there are at least three possibilities regarding the nature of this deficit. One possibility is that the dorsal stream itself is compromised. Here the system receives normal sensory input but fails to activate and/or generate appropriate temporal sequencing. On the other hand, most of the dorsal system itself may function normally, but poor sensory coding early in the network is sufficient to impair processing further through the dorsal stream. If this were the case, we would see the consequences of a normal system doing its best to cope with abnormal input. The third possibility is, of course, a combination of both. Demonstrating that normal and dyslexic readers perform the same on $\mathrm{M}(\mathrm{y})$ cell-mediated tasks allows us to reject the possibility that input into the dorsal stream is degraded. On the other hand, evidence for poor $\mathrm{M}(\mathrm{y})$ cell processing in dyslexia allows us at least to conclude that sensory information gaining access to the dorsal stream is impaired, but it does not preclude the possibility of general system impairment. How the dorsal stream in general contributes to reading and reading ability will be considered in the Discussion section.

This study is designed to evaluate the suggestion that visual sensitivity mediated by the dorsal visual system contributes to reading ability independently of nonverbal IQ. We will test this proposal in an experimental design utilizing the FD illusion as our index of magnocellular/dorsal activity as well as multiple measures of reading ability, including full-text reading and single-word recognition. Rather than a standard approach of matching readers on nonverbal IQ, here we use a sample of children in which both reading ability and IQ vary continuously. In addition, a second visual task is also used, which, although perceptually similar to the FD task and with identical task demands, has the crucial difference that it is unlikely to be mediated by the $\mathrm{M}(\mathrm{y})$ system.

If we can demonstrate that residual variance in reading can be attributed to FD visual sensitivity independently of both IQ and the non-FD visual control task, this would provide good evidence for a unique relationship between $\mathrm{M}(\mathrm{y})$ cell sensitivity and reading skill. 


\section{METHOD}

\section{Participants}

Participants were recruited from three local primary schools to take part in the study. Fifty-nine children participated in the study, however, 12 of these children were excluded as they were nonnative English speakers, and 4 children were excluded from the final analysis for failing to adhere to the experimental procedure. This resulted in 44 children (19 boys and 25 girls) contributing to the data. Participants were from a wide range of socioeconomic backgrounds. They ranged in age from 8 years 5 months to 12 years 3 months, with an average age of 10 years 6 months (see Table 1 for participant profiles). Consultation with specialist teachers indicated that none of the children had a history of neurological illness or emotional problems and that all had experienced normal educational opportunities. All children had normal or corrected-to-normal visual acuity and normal hearing.

The study had local ethics committee approval as well as additional approval from the department of education.

\section{Materials}

Psychometric measures. A broad range of cognitive and reading-related tests were administered to all the participants. All children were required to score within the normal range in nonverbal cognitive functioning as determined by Raven's Standard Progressive Matrices (Raven, 1989). A nonverbal IQ measure was chosen over a full-scale IQ measure for a couple reasons: First, we wanted an IQ measure that was independent of the children's language skills (see Bishop \& Snowling, 2004, for a discussion of this issue); second, we wanted to reduce the amount of overall testing time to minimize fatigue effects. Therefore we chose the Raven's test, which has been used elsewhere (e.g., Banai \& Ahissair, 2004; Pammer $\&$ Wheatley, 2001). However, given the degree to which different measures of cognitive performance load on reading ability, the results from this study cannot be generalized to all IQ measures and may be specific to nonverbal IQ.

Reading achievement was measured using the Neale Analysis of Reading Ability (NARA), an Australian standardized reading test consisting of passages of text of increasing difficulty. Scores include reading accuracy, comprehension, and speed (Neale, 1997). Performance on the reading accuracy and speed components of the NARA were combined to create a reading age score that shows the deviation of reading age from chronological age (in months). Thus the score indicates whether a participant's measured ability in reading is at a higher or lower achievement age than his or her expected chronological age. In addition to the standardized achievement tests, phonological and irregular word reading was also assessed. 
Rhyme discrimination. To assess the children's phonological skills, the children were given a rhyme discrimination task consisting of 26 word quadruplets (McDougall et al., 1994). On each trial the child was asked to identify the nonrhyming item from a set of four monosyllabic words, in which three words rhymed and one did not. As this was a test of rhyme discrimination, the discriminating feature always occurred on the final phoneme, for example, job, knock, rob, mob.

Phoneme deletion. The children's phonological skills were also assessed using a phoneme deletion task (McDougall et al., 1994). On each of the 24 trials the child heard a monosyllabic nonword. His or her task was to "take away" a specified sound from the nonword to make a real word. A recognizable word was always formed as a result of the sound deletion. For example, taking away the /b/ sound from the nonword barch produces the word arch.

Nonword and irregular word reading. The children's reading component skills were assessed by the Castles and Coltheart (1993) battery, a task comprising 30 regular words, 30 irregular words, and 30 pronounceable nonwords. Originally designed to determine reading disability subtypes, the irregular words and nonwords were used in this study to assess each child's phonological and irregular word reading skills, respectively(Talcott et al., 2002; Talcott et al., 1999; Witton et al., 1998). Regular words were included for the purpose of comparison with the irregular words. The irregular and regular words were matched on both frequency and imageability using the Medical Research Council Psycholinguistic Database (Coltheart, 1981), and each regular-irregular pair shared the same grammatical class and number of letters. Each word was printed in lowercase, 14-point Geneva font on white laminated cards. The child was instructed to read aloud what each word said. Children were told that they might find some of the words easy and some of them hard; however, it was stressed that they were not expected to know all of them. They were also told that some of the words were not real words but nonsense words, and they were encouraged to read these just like the other words. An example of a nonword was shown to the child before testing began. The order of presentation of the words was randomly determined for each participant.

\section{Visual Stimuli}

The experiment comprised two separate visual stimulus conditions: an FD and a non-FD condition. Each condition was presented separately to each eye. To test one eye at a time, standard reading glasses with one lens removed and the other lens covered with an opaque film were used. This method was used instead of a conventional eye patch to minimize dark adaptation in the covered eye. In the FD condition the stimuli were designed to be consistent with the Humphrey ${ }^{\circledR}$ Instru- 
ments FDT Visual Field Instrument ${ }^{\circledR}$ (Zeiss Humphrey Systems, California, USA), which has been used in previous studies (e.g., Buchholz \& McKone, 2004; Pammer et al., 2004; Pammer \& Wheatley, 2001). The stimuli consisted of low spatial frequency vertical sine wave gratings presented within a square aperture on a gray background. The 0.25 -cycles/deg gratings were modulated at $50-\mathrm{Hz}$ counterphase flicker; that is, the grating was flashed alternatively with another grating with a phase offset of $180^{\circ}$ to create the visual illusion of a grating that is twice the spatial frequency of the actual stimulus. In the non-FD condition a static, high spatial frequency grating of 0.5 cycles/deg was flickered alternatively with the gray background to create the same appearance as the FD gratings. Unlike the FD stimuli, the higher spatial frequency patterns in the non-FD stimuli were not the result of the illusion but instead were engineered to look like the product of the illusion. Refer to Figure 1 for a description of the stimuli used. All visual stimuli were presented on a BenQ 19-in. color monitor, driven by an Acer NVIDIA graphics card, with a screen refresh rate of $100 \mathrm{~Hz}$.

A trial sequence consisted of stimuli presented to one of five regions of the visual field measuring $35^{\circ}$ visual angle. Stimuli were presented centrally or in one of the four corners of the screen. The centrally presented stimuli were displayed at fixation, whereas the peripheral stimuli were displayed $14^{\circ}$ from fixation. Refer to Figure 2 for the experimental setup. For all conditions a small, black fixation square subtending $0.5^{\circ}$ was positioned in the center of the visual field and remained visible throughout the entire testing session. Each stimulus was a $10^{\circ}$ diameter square grating displayed for $720 \mathrm{~ms}$, including a 160-ms ramped onset and offset. The contrast of the stimulus at each location was manipulated according to a modified binary search threshold strategy (Tyrrell \& Owens, 1988). The modified binary search staircase

$A$

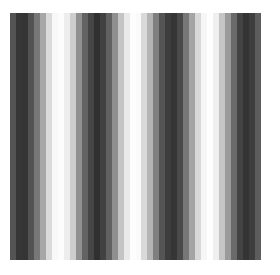

$B$

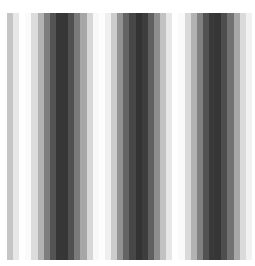

$C$

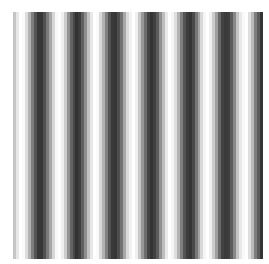

$D$

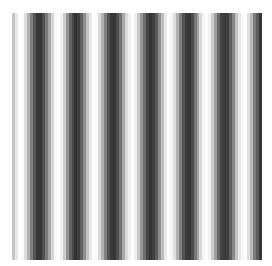

FIGURE 1 The physical stimulus A is alternated with stimulus B. The resulting visual illusion is $\mathrm{C}$, where the participant sees a grating at twice the spatial frequency of the two component gratings. Stimulus $C$ is the FD stimulus. Stimulus D is the non-FD stimulus, which was designed to look like the FD stimulus (C). However, stimulus $\mathrm{C}$ is a physical illusion resulting from a retinal nonlinearity in the visual system, whereas stimulus $\mathrm{D}$ is a simple sine wave grating constructed to be the same spatial frequency as the illusion in stimulus $\mathrm{C}$. 


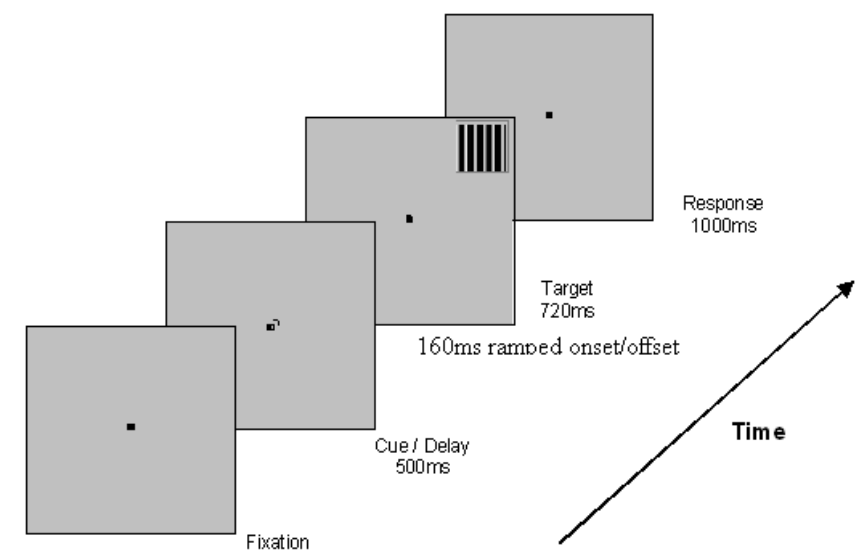

FIGURE 2 Schematic of the trial sequence. The arrow represents the sequence and timing of events. In this example the cue indicates a grating pattern in the top right corner of the display.

method determined contrast threshold by calculating the mean of the last upper and lower stimulus presentations, satisfying the staircase completion criteria consisting of four staircase reversals and a test range of less than $6 \mathrm{~dB}$. The mean luminance was $62.7 \mathrm{~cd} / \mathrm{m}^{2}$ for the FD stimuli and $48.1 \mathrm{~cd} / \mathrm{m}^{2}$ for the non-FD stimuli, with a mean background luminance of $37.5 \mathrm{~cd} / \mathrm{m}^{2}$. The range of possible threshold level values was between $0 \mathrm{~dB}(\sim 100 \%)$ maximum contrast (lowest sensitivity) and $40 \mathrm{~dB}(\sim 0 \%)$ minimum contrast (highest sensitivity).

One of the aims of the current experiment was to explore the generalizability of the FD illusion as a tool in reading research. Hence the stimuli were presented on a conventional computer monitor under normal lighting conditions, rather than on the commercial Humphrey Instruments FDT Visual Field Instrument. In addition, we wanted to include the control condition, which we were unable to do with the commercial product. However, the FD stimulus in the current experiment differed from previous experiments using the Humphrey Instruments FDT Visual Field Instrument by using only 5 testing locations rather than 17 . In this instance we were not interested in the diagnostic value of retinal perimetry, and we did not therefore consider it necessary to subject the children to an unnecessarily large number of trials by including the additional locations. The other primary difference between the current FD task and the commercial Humphrey Instruments FDT Visual Field Instrument is the counterphase flicker rate. Here we used a $50-\mathrm{Hz}$ counterphase flicker, rather than $25 \mathrm{~Hz}$. This was done simply because the illusion was far more stable at $50 \mathrm{~Hz}$ than at $25 \mathrm{~Hz}$. This is likely to be due to the less controlled stimulus 
environment, such as an interaction between raster rate, phosphor decay, and flicker frequency. A $50-\mathrm{Hz}$ flicker rate at a spatial frequency of 0.25 cycles $/ \mathrm{deg}$ still falls within the spatiotemporal parameters that produce the illusion originally identified by Kelly (1966). The important point here is that the illusion was reproducible, albeit using different spatiotemporal parameters, on a standard computer monitor using bespoke software. Nevertheless, because of these differences, pilot studies were conducted to determine the stability of the FD illusion in different observers. The results of this pilot study are reported in the Results section.

Central cues were presented in approximately $50 \%$ of trials (cued condition) in both the FD and non-FD conditions to indicate target location and thus aid in the detection of the gratings. The cue consisted of a small black arrow subtending $0.7^{\circ}$ and presented $0.3^{\circ}$ from fixation pointing to the location of a surround target. A small black cross subtending $0.7^{\circ}$ presented at fixation was used to alert the participant that the target would be presented in the center of the screen. Each cue was presented for $500 \mathrm{~ms}$, followed by presentation of the target. The maximum time allowed to respond was 1,000 ms (see Figure 2). No invalid cues were presented. This cueing condition was part of a larger ongoing project and as such is not discussed further here.

Two kinds of catch trial were included in the trials to ensure that the children understood the directions correctly and were not simply responding indiscriminately to the stimuli. Ten percent of the trials were negative catch trials, in which the target was not presented and participants did not have to respond. A response to a negative catch trial was an indication that a child was responding whether they saw a display or not. To discourage responses to the cue and not the stimulus itself, negative catch trials that consisted of the presentation of a cue without a target were also included. Positive catch trials (10\% of trials), in which a stimulus was presented at the maximum possible contrast level, were also included to determine whether the child could actually see the stimulus and to serve as a general vigilance measure.

\section{Procedure}

The children were tested individually in a quiet, well-lit room, with the windows covered to control for natural light variation. Each child was seated comfortably in front of a computer monitor with his or her head positioned on a chin rest so that the eye-screen distance was $34 \mathrm{~cm}$. The child was given a description of the display, and each child completed a practice session, consisting of the grating pattern occurring randomly in all possible locations, until the child felt confident with the task. It was emphasized to the child that he or she must continue to keep his or her eyes on the fixation point throughout the entire testing sequence. Eye movements were monitored by means of a system composed of a video camera connected to an eye-tracking program. Any eye movements larger than $10^{\circ}$ were detected by the 
Video Trace ${ }^{\circledR}$ System, and the corresponding trial was discarded and replaced. Calibration of the measurements were performed directly before each stimulus block by having the participant fixate 20 small black dots plotted onto the central $35^{\circ}$ of the visual field.

The participants' task was to press the space bar of the computer keyboard once they detected the stimulus, and their reaction times and responses were recorded by the computer. Participants were instructed to respond as quickly as possible whenever a target was detected at any of the five locations. The testing sequence for each eye took approximately $5 \mathrm{~min}$, and the total testing time, including the psychometric tests, was approximately $1.5 \mathrm{hr}$ for each participant, which was broken into two sessions to avoid the effects of fatigue and inattention. The order of all tests was randomized over all participants, and the children were encouraged to take regular breaks.

\section{RESULTS}

\section{Validity of FD Measure}

Studies conducted elsewhere have used the commercial Humphrey Instruments FDT Visual Field Instrument (e.g., Buchholz \& McKone, 2004; Pammer et al., 2004; Pammer \& Wheatley, 2001). To establish that the reconstruction of the FD stimuli on a standard PC does in fact produce the FD illusion, a pilot study was conducted. There were four experienced observers, three naive observers, and one child ( 8 years old, to be consistent with the youngest of the anticipated participants). These pilot participants were asked to match the FD stimulus used in the main study (stimulus $C$ in Figure 1) with either the non-FD stimulus (stimulus D in Figure 1) or with the component grating that would be seen if the FD illusion was not present (stimulus A or B in Figure 1). Participants were given between 30 and 200 trials, and all participants reported seeing the FD illusion (i.e., C of Figure 1, rather than $\mathrm{A}$ or $\mathrm{B}$ ) on $100 \%$ of trials. This would suggest that like the commercial product, the FD stimulus presented in this experiment is a reliable reproduction of the FD illusion. Moreover, we obtained a correlation between FD sensitivity and reading ability $(r=.6)$ for our full sample, which is consistent with other studies reporting a correlation of $r=.4$ (Pammer et al., 2004) and $r=.6$ (Pammer \& Wheatley, 2000) using the Humphrey Instruments FDT Visual Field Instrument.

Table 1 summarizes the performance of participants on a range of cognitive and reading-related tasks. The dependent variable for the FD and non-FD tasks was contrast threshold for stimulus detection. Data concerning practice trials were not included in the analysis.

Screening analyses were conducted to determine the degree to which individual variability on the following variables might influence the visual sensitivity results. 
TABLE 1

Reading and Cognitive Tests Descriptive Data

\begin{tabular}{|c|c|c|c|c|c|}
\hline \multirow[b]{2}{*}{ Measures } & \multicolumn{2}{|c|}{ Normal $^{\text {a }}$} & \multicolumn{2}{|c|}{ Dyslexic $^{\mathrm{b}}$} & \multirow[b]{2}{*}{$t$ Test $(d f=42)$} \\
\hline & $M$ & $S D$ & $M$ & $S D$ & \\
\hline Raven's IQ & 117.82 & 10.343 & 103.34 & 10.601 & $4.550 *$ \\
\hline NARA (months) & 15.84 & 14.996 & -32.84 & 14.377 & $12.230^{*}$ \\
\hline Phoneme deletion & 19.80 & 3.175 & 11.89 & 5.446 & $5.996^{*}$ \\
\hline Rhyme discrimination & 21.56 & 4.174 & 15.32 & 3.845 & $5.083^{*}$ \\
\hline Regular words & 29.16 & 1.405 & 22.58 & 6.526 & $6.208^{*}$ \\
\hline Irregular words & 21.76 & 3.756 & 13.53 & 5.651 & $5.710 *$ \\
\hline Nonwords & 26.80 & 3.136 & 16.58 & 7.018 & $6.504 *$ \\
\hline
\end{tabular}

Note. Neale Analysis of Reading Ability (NARA) scores indicate deviation (in months) from expected reading level for their chronological age.

${ }^{\mathrm{a}} n=25 . \mathrm{b}_{n}=19$.

$* p<.001$.

The screening variables included fixation stability, stimulus location, age, left/right eye, positive and negative catch trials, and visual field. None of these variables were correlated with reading ability, visual sensitivity (both FD and non-FD sensitivity), or nonverbal IQ. Moreover, none of these factors predicted reading ability when entered into a regression analysis. This suggests that variability in vigilance (as indicated by eye movements or catch trials) or other visual factors (such as left/right eye or stimulus location) were unlikely to be contributing factors here.

Correlational analyses were further conducted to explore the relationship between the variables. IQ correlated .65 with NARA reading ability. Tasks designed to tap into phonological skills (rhyme discrimination, phoneme deletion, and nonword reading) were significantly correlated at $p<.001, r=.68$ for nonword reading and rhyme, $r=.59$ for rhyme and phoneme deletion, and $r=.81$ for phoneme deletion and nonword reading. These scores were therefore converted to $z$ scores and combined into a single factor measuring phonological skill. Cronbach's alpha for these data was calculated to be .87, suggesting an acceptable degree of reliability between the three estimates of phonological skill.

Bivariate correlations were calculated to assess the relationship between the visual and cognitive tasks. Inspection of Table 2 indicates that age did not appear to influence the data and that the two visual tasks were significantly correlated with each other. As expected, both the FD and non-FD tasks were also significantly correlated with reading ability $(r=.66, p<.01$ and $r=.35, p<.05$, respectively). Similarly, both FD and non-FD were correlated with irregular word reading $(r=.6, p<$ .01 and $r=.36, p<.05$, respectively), and FD was significantly correlated with phonological skill $(r=.46, p<.01)$, but there was no correlation between non-FD and phonological skill. 
TABLE 2

Correlation Matrix Between All Reading and Visual Tasks

\begin{tabular}{lcccccc}
\hline & $I Q$ & $R A$ & $A G E$ & $O A$ & $P S$ & $F D$ \\
\hline IQ & & & & & & \\
RA & $.635^{* *}$ & & & & & \\
AGE & -.102 & -.145 & & & & \\
IWR & $.363^{*}$ & $.704^{* *}$ & .231 & & & \\
PS & $.645^{* *}$ & $.779^{* *}$ & .200 & $.626^{* *}$ & & $.606^{* *}$ \\
FD & $.544^{* *}$ & $.660^{* *}$ & -.059 & $.606 *$ & $.459 * *$ \\
Non-FD & .097 & $.351^{*}$ & .071 & $.363^{*}$ & .081 & $.542^{* *}$ \\
\hline
\end{tabular}

Note. IQ = nonverbal IQ (Raven's); RA = reading ability (Neale Analysis of Reading Ability); $\mathrm{AGE}=$ chronological age; IWR = irregular word reading; PS = phonological skill (phoneme deletion, rhyme discrimination, and nonword reading); FD = frequency doubling illusion sensitivity; Non-FD = non-FD sensitivity.

$* p<.05 . * * p<.01$.

Because nonverbal IQ correlated with both reading ability and the FD task, it is possible that sensitivity to the FD stimuli might simply have been due to nonverbal IQ differences in the readers, as would be expected if general task demands accounted for differences in visual sensitivity. However, partial correlations between reading ability and FD sensitivity, controlling for nonverbal IQ, suggest that IQ does not fully explain this relationship $(r=.49, p<.001$ for the correlation between reading ability and FD sensitivity). Therefore regression analyses were used to explore the contribution of the FD task to reading ability, partialling out a number of other variables.

\section{Relationship Between Reading Skills and FD Stimuli}

Hierarchical multiple regression analysis was used to determine whether the FD task could predict component reading skills and reading ability (see Table 3). As nonverbal IQ and non-FD sensitivity correlated significantly with FD sensitivity, they were the first variables entered in each model. In the first regression, IQ, non-FD sensitivity, and FD sensitivity were used to predict reading ability (NARA). Results show that although nonverbal IQ was the best predictor of reading ability, FD sensitivity nevertheless significantly predicted reading ability over and above nonverbal IQ and non-FD sensitivity, $t(42)=2.385, p<.05$, accounting for $6 \%$ of unique variance. Two further regression analyses were conducted to explore the possibility that FD sensitivity could also explain unique portions of the variance associated with the component skills of reading ability, namely, phonological and orthographic processing. Thus the second and third regressions used nonverbal IQ, non-FD sensitivity, and FD sensitivity to predict phonological skill and irregular word reading, respectively. 
TABLE 3

Summary of the Regression Models in Which IQ, FD, and Non-FD

Sensitivity Were Entered as Predictor Variables of Various Aspects of Reading Performance

\begin{tabular}{|c|c|c|c|c|c|c|c|c|c|}
\hline \multirow[b]{2}{*}{ Model } & \multicolumn{3}{|c|}{$N A R A$} & \multicolumn{3}{|c|}{$P S$} & \multicolumn{3}{|c|}{$I W R$} \\
\hline & $\beta$ & $p$ & $s r^{2}$ & $\beta$ & $p$ & $s r^{2}$ & $\beta$ & $p$ & $s r^{2}$ \\
\hline 1. IQ & .606 & .000 & .367 & .643 & .000 & .412 & .346 & .016 & .119 \\
\hline \multirow[t]{2}{*}{ Non-FD } & .293 & .013 & .085 & .048 & .691 & .002 & .335 & .019 & .112 \\
\hline & \multicolumn{3}{|c|}{$R^{2}=.488, p<.000$} & \multicolumn{3}{|c|}{$R^{2}=.419, p<.000$} & \multicolumn{3}{|c|}{$R^{2}=.244, p<.01$} \\
\hline 2. IQ & .422 & .003 & .116 & .542 & .001 & .184 & .071 & .662 & .003 \\
\hline Non-FD & .109 & .410 & .008 & .055 & .722 & .001 & .054 & .741 & .002 \\
\hline \multirow[t]{2}{*}{ FD } & .371 & .022 & .064 & .197 & .284 & .017 & .539 & .008 & .127 \\
\hline & \multicolumn{3}{|c|}{$R^{2} \Delta=.064, p<.05$} & \multicolumn{3}{|c|}{$R^{2} \Delta=.017, p=.284$} & \multicolumn{3}{|c|}{$R^{2} \Delta=.127, p<.01$} \\
\hline
\end{tabular}

Note. $R^{2} \Delta=\mathrm{R}^{2}$ change for the respective models; $\mathrm{sr}^{2}=$ semipartial correlation; IQ = nonverbal IQ (Raven's); FD = frequency doubling illusion sensitivity; Non-FD = non-FD sensitivity; NARA = Neale Analysis of Reading Ability; PS = phonological skills (phoneme deletion, rhyme discrimination, and nonword reading); IWR = irregular word reading.

Results from the second regression analysis, with phonological skill as the dependent variable, demonstrate that nonverbal IQ is the sole predictor of phonological skill, $t(42)=3.564, p<.001$, accounting for $18 \%$ of unique variance (see Table 3). This is intriguing given the strong correlation between FD and phonological skill in the correlation matrix. However, the relationship between phonological skill and FD sensitivity dropped from being significantly correlated, $r(43)=.459$, $p<.01$, in the initial correlation to nonsignificant, $r(40)=.173, p=.272$, in the partial correlation, controlling for nonverbal IQ. This suggests that the correlation between phonological skill and FD sensitivity may indeed be due to the shared requirements of cognitive functioning.

The third regression analysis was the same as the other regression analyses, this time using irregular word reading as the dependent variable, and indicates that in Model 1, both nonverbal IQ and non-FD sensitivity were significant predictors of irregular word reading; however, when FD sensitivity was put into the model, only FD sensitivity was left to significantly predict irregular word reading, $t(42)=$ $2.808, p<.01$, accounting for $13 \%$ of unique variance.

As FD sensitivity was found to be a significant predictor of both irregular word reading and reading ability (reading speed and accuracy), it was necessary to conduct another regression analysis to determine whether FD sensitivity could predict reading ability over and above irregular word reading, still controlling for nonverbal IQ. Both IQ and FD sensitivity were entered into the regression first, followed by irregular word reading. Inspection of Table 4 indicates that although FD sensitivity significantly predicted reading ability over and above IQ, $t(42)=3.628, p<$ 
TABLE 4

Summary of the Models in Which the IQ, FD Sensitivity and Irregular Word Reading Ability Were Entered as Predictor Variables of Reading Ability

\begin{tabular}{lccc}
\hline & & $R A$ & \\
\cline { 2 - 4 } Model & $\beta$ & $p$ & $s r^{2}$ \\
\hline 1. IQ & .388 & .004 & .107 \\
FD & .457 & .001 & .148 \\
2. IQ & .365 & .002 & .094 \\
FD & .194 & .131 & .019 \\
IWR & .453 & .000 & .129 \\
\hline
\end{tabular}

Note. $\quad R^{2} \Delta=.550, p<.000$ for Model 1 and $R^{2} \Delta=.130, p<.000$ for Model $2 . s r^{2}=$ semipartial correlation; RA = reading ability (Neale Analysis of Reading Ability); IQ = nonverbal IQ (Raven's); $\mathrm{FD}=$ FD sensitivity; IWR $=$ IWR irregular word reading.

.001 , when irregular word reading was entered into the regression, FD sensitivity was no longer significant, $t(42)=1.541, p=.131$. This indicates that orthographic skill, as measured by irregular word reading, may partially mediate the relationship between FD sensitivity and reading ability.

\section{DISCUSSION}

In this study we found that nonverbal IQ was always the best predictor of reading ability, as would be expected when IQ and reading ability have a strong positive correlation. However, we nevertheless found that visual sensitivity to the FD illusion explains an additional, independent portion of the variance associated with reading ability. This is consistent with previous studies using group analyses that demonstrated that poor readers are significantly less sensitive to the FD illusion than normal readers (Buchholz \& McKone, 2004; Pammer et al., 2004; Pammer \& Wheatley, 2001). Moreover, the evidence that the control non-FD task does not make an independent contribution to reading ability when considered with FD sensitivity enables us to dismiss the counterproposal that poorer readers are likely to be poorer at all visual psychophysical tasks.

In this study the FD and non-FD stimuli were designed to look similar, and informal observation of the children suggested they were unaware that two different visual stimuli were presented. However, no special effort was made to match the two stimuli at an individual level on their physical properties. This is likely to provide an explanation for the observation that all children found the FD stimuli harder to see than the non-FD stimuli. However, FD sensitivity was significantly correlated with IQ, whereas non-FD sensitivity was not. Given the identical task demands in the FD and non-FD tasks, this pattern is likely to be the result of the 
strong correlation between reading ability and IQ; that is, the children with the lowest IQs also demonstrated the poorest reading skills. Thus the correlation between IQ and FD is most likely mediated by poor reading, not poor IQ. Indeed, when we partial out reading ability from the correlation between FD and IQ, the correlation becomes nonsignificant (the correlation drops from $r=.54, p<.01$ to $r$ $=.23, p>.05$ ). A significant correlation was also found between FD and non-FD sensitivity. This is not unexpected given that the non-FD control task was designed to be identical to the FD task with regard to task structure and demands. However, because of the temporal flicker associated with the non-FD tasks, it is possible that the non-FD task recruited magnocellular resources. Nevertheless, the non-FD task consisted of spatiotemporal qualities outside those specified as contributing to the illusion (Kelly, 1966), and there was no reported experience of a frequency-doubled illusion with the non-FD task. This suggests that although the non-FD task may involve magnocellular processing, it is unlikely to be drawing explicitly on $\mathrm{M}(\mathrm{y})$ cell resources. Indeed, non-FD sensitivity significantly predicted reading ability when entered into the regression model along with nonverbal IQ. This correlation dropped to nonsignificance when the FD task was entered. If the non-FD task was drawing on magnocellular resources, then at the very least, the methodological implication of this is that sensitivity to the FD illusion is a better visual technique for predicting reading ability than magnocellular-mediated visual tasks in general. The theoretical implication may be that the primary contribution of visual sensitivity to reading ability may be at the very front end of the magnocellular pathway, originating at the level of the retina. However, this was the first study to provide a control condition designed to be as close as possible to the FD stimulus, and as such, we have little data to describe how the actual perceptual experience of these stimuli varies with reading ability, particularly at low contrast. Therefore, although we consider it unlikely, it is possible that there may be otherwise unrecognized differences between the FD and non-FD stimuli.

This study was designed to assess the contribution of visual sensitivity to reading ability, independently of nonverbal IQ, in a situation in which nonverbal IQ would be expected to correlate with reading ability. This differs from previous studies (e.g., Buchholz \& McKone, 2004; Cornelissen, Richardson, Mason, Fowler, \& Stein, 1995; Facoetti, Paganoni, \& Lorusso, 2000) because nonverbal IQ was allowed to vary freely within the same sample rather than simply being artificially matched across good and poor reader groups. If there is any validity to the notion that visual processing difficulties may be causal in reading difficulty, then it must be clearly demonstrated that visual difficulties are not merely epiphenomenal to a cognitive deficit. Although reading ability was found to be correlated with IQ, FD sensitivity remained correlated with reading ability, even when IQ was controlled for. The contribution remained after non-FD sensitivity was entered into the regression equation. This suggests that although some of the differences between 
good and poor readers in FD sensitivity may be related to nonverbal IQ, FD sensitivity has a unique relationship to reading ability.

As FD sensitivity was demonstrated to be a significant predictor of reading ability, the relationship between FD sensitivity and component reading skills was also investigated. FD sensitivity was correlated with measures of phonological processing and irregular word reading. It was therefore interesting to see whether sensitivity to seeing FD stimuli could predict the component reading skills independently of IQ. Results demonstrated that sensitivity to seeing FD stimuli did not predict phonological processing when IQ was controlled for. This is not surprising given that phonological skills are suggested to be a higher order integration process and that FD sensitivity is proposed to be a measure of low-level sensory processing. It is an open question as to whether the reading of irregular words might be more highly related to visual sensitivity (Snowling, 2001; Talcott et al., 2002; Vellutino et al., 2004). FD sensitivity is proposed to be a measure of low-level sensory processing (Buchholz \& McKone, 2004; Pammer et al., 2004; Pammer \& Wheatley, 2001). Indeed, FD sensitivity predicted $13 \%$ of variance in irregular word reading, over and above IQ and non-FD sensitivity. Moreover, although FD sensitivity was initially found to be a significant predictor of reading ability, once orthographic skill (irregular word reading) was entered into the model, FD sensitivity was no longer a significant predictor of reading ability. Therefore the mediating relationship between FD sensitivity, orthographic skill, and reading ability provides evidence to suggest that the visual deficits observed in dyslexic readers may be linked to their reading impairments through some common process involved in both reading passages and reading irregular words.

What might this common process be? It has been demonstrated elsewhere that visual sensitivity is important for different aspects of orthographic processing such as position encoding (e.g., Cornelissen et al., 1998; Pammer et al., 2004). It has been suggested (Vidyasagar, 1999, 2001, 2005; Vidyasagar \& Pammer, 1999) that the relationship between accurate position encoding and magnocellular functioning may arise from the role the magnocellular pathway plays in the preattentive control of spatial selection; such effective magnocellular processing may be necessary for accurate spatial sequencing to allow for the visual construction of lines in letters and letters within words. However, the question arises as to how a deficit in early spatial coding mechanisms might impair the lexical route more so than the phonological route. Surely, reading all three categories of words elicits the same basic visual mechanisms? One possibility may lie in the differences in cortical activation underlying lexicality. Preattentive processing in the dorsal stream provides information to the ventral stream about salient spatial selection to facilitate feature extraction and shape processing (Vidyasagar, 1999). Indeed, the visual word form area identified by Cohen and colleagues (e.g., Cohen et al., 2000; Cohen et al., 2002) is positioned at the posterior part of the left fusiform gyrus (FF), consistent with a posterior termination of the ventral stream. The visual word form area ap- 
pears to be involved in the elaboration of shape and position information in visual word forms. However, processing of lexical information may occur more anteriorly on the FF. Binder, Medler, Desai, Conant, and Liebenthal (2005) demonstrated that the anterior FF (FFa) is involved in processing irregular and regular words but not nonwords. Similarly, Price et al. (2003) described patient JH who has semantic dementia as a result of cortical atrophy in the FFa. JH could read nonwords and regular words but found it very difficult in comparison to read irregular words. This part of the inferior temporal cortex also receives direct attentional modulation that is likely to be mediated by the dorsal pathway (e.g., Chelazzi, Miller, Duncan, \& Desimone, 1993; Motter, 1994; Reynolds, Pasternak, \& Desimone, 2000; Sato, 1988).

One possibility, therefore, is that the anterior portion of the FF is involved in the processing of known word forms, as would be used for recognizing regular words, and must be required for recognizing irregular words. Thus when presented with an irregular word (for example), the flow of information through the $\mathrm{FFa}$ subnetwork is designed to extract a recognizable word. When a recognizable word is not quickly encountered, no representations are activated, and the network recruits other cortical processes, such as the grapheme-to-phoneme correspondence rules assembly, at other parts of the reading network. This model is supported by the evidence that response latencies are longer for nonwords compared to irregular words as well as regular words (Binder et al., 2005) and that the FFa is involved in the recognition of known objects, compared to the posterior FF, which appears to be involved in feature extraction and assembly (Bar et al., 2001). Therefore the dorsal stream may be more influential in regular and irregular word reading because activity in the FFa (putatively, the anterior portion of the ventral stream), intrinsic to direct lexical access, is more tightly coupled in terms of time and direct connectivity than higher cortical processes mediated, for example, by the left frontal operculum (e.g., Fiez, Balota, Raichle, \& Petersen, 1999; Hagoort et al., 1999; Xu et al., 2001; see Mechelli, Gorno-Tempini, \& Price, 2003), which are likely to be recruited for grapheme-to-phoneme correspondence assembly in reading nonwords. This proposal is highly tentative at this stage, and clearly, further research is required to judge the validity of the model and to assess the association (if any) to phonological encoding.

These results therefore challenge the idea that visual impairments in poorer readers are simply the consequences of increased cognitive load or poor vigilance. Although evidence for a phonological processing deficit in dyslexia is strong, it is now clear that proficient reading requires the contribution of both orthographic and phonological processes (Stein, 2001; Stein \& Walsh, 1997; Talcott et al., 2002; Talcott et al., 2000). Therefore visual deficits may lead to a unique impairment in orthographic skill, which in turn leads to a decrease in reading performance.

This study provides good evidence to suggest that reading proficiency depends on the contribution of the visual dorsal system to enable the acquisition of reliable or- 
thographic representations. However, clearly, orthographic processing is not the sole contributor to the reading process as phonological processing undeniably plays a vital contributing role. Therefore further research is needed to determine not only the nature of the visual deficit but also how the interplay of phonological and orthographic processing skills contributes to the reading process. Indeed, knowing this may lead to a greater understanding of whether visual skills are causally related to reading ability or are simply neurological markers of a phonological deficit.

\section{ACKNOWLEDGMENTS}

We are grateful to Andy Thomson for writing the programs, and his consistent and patient technical assistance. Thanks also to the three participating schools. We are also grateful to three anonymous reviewers whose comments have made this an altogether better article.

\section{REFERENCES}

Amitay, S., Ben-Yehudah, G., Banai, K., \& Ahissar, M. (2002). Disabled readers suffer from visual and auditory impairments but not from a specific magnocellular deficit. Brain, 125, 2272-2285.

Banai, K., \& Ahissar, M. (2004). Poor frequency discrimination probes dyslexics with particularly impaired working memory. Audiology and Neuro-Otology, 9, 328-340.

Bar, M., Tootell, R., Schacter, D., Greve, D., Fischl, B., Mendola, J., et al. (2001). Cortical mechanisms specific to explicit visual object recognition. Neuron, 29, 529-535.

Bedford, S., Maddess, T., Rose, K., \& James, A. (1997). Correlations between observability of the spatial frequency doubling illusion and a multi-region pattern electroretingram. Australian and New Zealand Journal of Ophthalmology, 25, S91-S93.

Benardete, E. A., Kaplan, E., \& Knight, B. W. (1992). Contrast gain control in the primate retina: P cells are not X-like, some M cells are. Visual Neuroscience, 8, 483-486.

Ben-Yehudah, G., Sackett, E., Malchi-Ginzberg, L., \& Ahissar, M. (2001). Impaired temporal contrast sensitivity in dyslexics is specific to retain-and-compare paradigms. Brain, 124, 1381-1395.

Binder, J., Medler, D., Desai, R., Conant, L., \& Liebenthal, E. (2005). Some neurophysiological constraints on models of word naming. Neuroimage, 27, 677-693.

Bishop, D., \& Snowling, M. (2004). Developmental dyslexia and specific language impairment: Same or different? Psychological Bulletin, 130, 858-886.

Buchholz, J., \& McKone, E. (2004). Adults with dyslexia show deficits on spatial frequency doubling and visual attention tasks. Dyslexia, 10, 24-43.

Casco, C. (1993). Visual processing of static and dynamic information in disabled readers. Clinical Vision Sciences, 8, 461-471.

Castles, A., \& Coltheart, M. (1993). Varieties of developmental dyslexia. Cognition, 47, 149-180.

Chelazzi, L., Miller, E., Duncan, J., \& Desimone, R. (1993). A neural basis for visual search in inferior temporal cortex. Nature, 363, 307-345.

Cohen, L., Dehaene, S., Naccache, L., Lehericy, S., Dehaene-Lambertz, G., Henaff, M., et al. (2000). The visual word form area: Spatial and temporal characterisation of an initial stage of reading in normal subjects and posterior split-brain patients. Brain, 123, 291-307. 
Cohen, L., Lehericy, S., Chochon, F., Lemer, C., Rivaud, S., \& Dehaene, S. (2002). Language specific tuning of the visual cortex? Functional properties of the visual word form area. Brain, 125, 1054-1069.

Coltheart, M. (1981). The MRC Psycholinguistic Database. Quarterly Journal of Experimental Psychology, 33A, 497-508.

Cornelissen, P., \& Hansen, P. (1998). Motion detection, letter position encoding, and single word reading. Annals of Dyslexia, 48, 155-188.

Cornelissen, P. L., Hansen, P. C., Gilchrist, I., Cormack, F., Essex, J., \& Frankish, C. (1998). Coherent motion detection and letter position encoding. Vision Research, 38, 2181-2191.

Cornelissen, P. L., Richardson, A., Mason, A., Fowler, S., \& Stein, J. (1995). Contrast sensitivity and coherent motion detection measured at photopic luminance levels in dyslexics and controls. Vision Research, 35, 1483-1494.

Demb, J. B., Boynton, G. M., Best, M., \& Heeger, D. J. (1998). Psychophysical evidence for a magnocellular pathway deficit in dyslexia. Vision Research, 38, 1555-1559.

Eden, G. F., Van Meter, J. W., Rumsey, J. M., Maisog, J. M., Woods, R. P., \& Zeffiro, T. A. (1996). Abnormal processing of visual motion in dyslexia revealed by functional brain imaging. Nature, 382 , 66-69.

Facoetti, A., Paganoni, P., \& Lorusso, M. L. (2000). The spatial distribution of visual attention in developmental dyslexia. Experimental Brain Research, 132, 531-538.

Fiez, J., Balota, D., Raichle, M., \& Petersen, S. (1999). Effects of lexicality, frequency, and spelling-to-sound consistency on the functional anatomy of reading. Neuron, 24, 205-218.

Francks, C., MacPhie, I. L., \& Maonaco, A. P. (2002). The genetic basis of dyslexia. Neurology, 1, 483-490.

Galaburda, A. M., \& Livingstone, M. S. (1993). Physiological evidence for a magnocellular defect in developmental dyslexia. Annals of the New York Academy of Sciences, 682, 70-82.

Hagoort, P., Indefrey, P., Brown, C., Herzog, H., Steinmetz, H., Seitz, R. J., et al. (1999). The neural circuitry involved in the reading of German words and pseudowords: A PET study. Journal of Cognitive Neuroscience, 11, 383-398.

Hayduk, S., Bruck, M., \& Cavanagh, P. (1996). Low-level visual processing skills of adults and children with dyslexia. Cognitive Neuropsychology, 13, 975-1015.

Hogben, J. (1996). A plea for purity. The Australian Journal of Psychology, 48, 172-177.

Hulslander, J., Talcott, J. B., Witton, C., DeFries, J., Pennington, B. F., Wadsworth, S., et al. (2004). Sensory processing, reading, IQ, and attention. Journal of Experimental Child Psychology, 88, 274-295.

Johnson, C., \& Shapiro, L. (1989). A comparison of MOBS (modified binary search) and staircase test procedures in automated perimetry. In Noninvasive Assessment of the Visual System Technical Digest Series (pp. 84-87). Washington, DC: Optical Society of America Press.

Keen, A., \& Lovegrove, W. J. (2000). Transient deficit hypothesis and dyslexia: Examination of whole-parts relationship, retinal sensitivity, and spatial and temporal frequencies. Vision Research, 40, 705-715.

Kelly, D. H. (1966). Frequency doubling in visual responses. Journal of the Optical Society of America, $56,1628-1633$.

Kelly, D. H. (1981). Nonlinear responses to flickering sinusoidal gratings. Journal of the Optical Society of America, 71, 1051-1055.

Kinsey, K., Rose, M., Hansen, P. C., Richardson, A., \& Stein, J. F. (2004). Magnocellular mediated visual-spatial attention and reading ability. Neuroreport, 14, 2215-2218.

Lovegrove, W. J., Martin, F., Bowling, A., Blackwood, M., Badcock, D., \& Paxton, S. (1982). Contrast sensitivity functions and specific reading disability. Neuropsychologia, 20, 309-315.

Maddess, T., Goldberg, I., Dobinson, J., Wine, S., Welsh, A. H., \& James, A. C. (1999). Testing for glaucoma with the spatial frequency doubling illusion. Vision Research, 39, 4258-4273. 
Maddess, T., \& Henry, G. H. (1992). Performance of nonlinear visual units in ocular hypertension and glaucoma. Clinical Vision Sciences, 7, 371-383.

McDougall, S., Hulme, C., Ellis, A., \& Monk, A. (1994). Learning to read: The role of short-term memory and phonological skills. Journal of Experimental Child Psychology, 58, 112-133.

Mechelli, A., Gorno-Tempini, M., \& Price, C. (2003). Neuroimaging studies of word and pseudoword reading: Consistencies, inconsistencies, and limitations. Journal of Cognitive Neuroscience, 15, 260-271.

Motter, B. (1994). Neural correlates of feature selective memory and pop-out in extrastriate area V4. Journal of Neuroscience, 14, 2190-2199.

Neale, M. (1997). Neale analysis of reading ability. Windsor, CT: NFER-Nelson.

Olson, R., \& Datta, H. (2002). Visual temporal processing in reading-disabled and normal twins. Reading and Writing: An Interdisciplinary Journal, 15, 127-149.

Pammer, K., Lavis, R., \& Cornelissen, P. L. (2004). Visual encoding mechanisms and their relationship to text presentation preference. Dyslexia, 10, 77-94.

Pammer, K., \& Wheatley, C. (2001). Isolating the M(y)-cell response in dyslexia using the spatial frequency doubling illusion. Vision Research, 41, 2139-2147.

Price, C., Gorno-Tempini., M., Graham, K., Biggio, N., Mechelli, A., Patterson, K., et al. (2003). Normal and pathological reading: Converging data from lesion and neuroimaging studies. Neuroimage, 20, S30-S41.

Ramus, F., Rosen, S., Dakin, S. C., Day, B. L., Castellote, J. M., White, S., et al. (2003). Theories of developmental dyslexia: Insights from a multiple case study of dyslexic adults. Brain, 126, 841-865.

Raven, J. C. (1989). Standard progressive matrices. Melbourne: Australian Council for Educational Research.

Rayner, K., Pollatsek, A., \& Bilsky, A. B. (1995). Can a temporal processing deficit account for dyslexia? Psychonomic Bulletin and Review, 2, 501-507.

Reynolds, J., Pasternak, T., \& Desimone, R. (2000). Attention increases sensitivity of V4 neurons. Neuron, 26, 703-714.

Sato, T. (1988). Effects of attention and stimulus interaction on visual responses of inferior temporal neurons in macaque. Journal of Neurophysiology, 60, 344-364.

Shaywitz, S. E. (1998). Dyslexia. New England Journal of Medicine, 338, 307-312.

Skottun, B. C. (2000). On the conflicting support for the magnocellular-deficit theory of dyslexia. Trends in Cognitive Sciences, 4, 211-212.

Skoyles, J., \& Skottun, B. C. (2004). On the prevalence of magnocellular deficits in the visual system of non-dyslexic individuals. Brain and Language, 88, 79-82.

Snowling, M. J. (2001). From language to reading and dyslexia. Dyslexia, 7, 37-46.

Stein, J. F. (2001). The magnocellular theory of developmental dyslexia. Dyslexia, 7, 12-36.

Stein, J. F. (2003). Visual motion sensitivity and reading. Neuropsychologia, 41, 1785-1793.

Stein, J. F., \& Walsh, V. (1997). To see but not to read: The magnocellular theory of dyslexia. Trends in Neuroscience, 20,147-152.

Stuart, G., McAnally, K., \& Castles, A. (2001). Can contrast sensitivity functions in dyslexics be explained by inattention rather than a magnocellular deficit? Vision Research, 41, 3205-3211.

Talcott, J. B., Witton, C., Hebb, G. S., Stoodley, C. J., Westwood, E. A., France, S. J., et al. (2002). On the relationship between dynamic visual and auditory processing and literacy skills: Results from a large primary-school study. Dyslexia, 8, 204-225.

Talcott, J. B., Witton, C., McLean, M. F., Hansen, P. C., Rees, A., \& Green, G. G. R. (2000). Dynamic sensory sensitivity and children's word decoding skills. Proceedings of the National Academy of Sciences, 97, 2952-2957.

Talcott, J. B., Witton, C., McClean, M., Hansen, P. C., Rees, A., Green, G. G. R., et al. (1999). Can sensitivity to auditory frequency modulation predict children's phonological and reading skills? Neuroreport, 10, 2045-2050. 
Tyler, C. W. (1974). Observations on spatial-frequency doubling. Perception, 3, 81-86.

Tyrrell, R., \& Owens, A. (1988). A rapid technique to assess the resting states of the eyes and other threshold phenomena: The Modified Binary Search (MOBS). Behavior Research Methods, Instruments and Computers, 20, 137-141.

Vanni, S., Dojat, M., Warnking, J., Delon-Martin, C., Segebarth, C., \& Bullier, J. (2004). Timing of interactions across the visual field in the human cortex. Neuroimage, 21, 818-828.

Vellutino, F. R., Fletcher, J. M., Snowling, M. J., \& Scanlon, D. M. (2004). Specific reading disability (dyslexia): What have we learned in the past four decades? Journal of Child Psychology and Psychiatry, 45, 2-40.

Vidyasagar, T. R. (1999). A neuronal model of attentional spotlight: Parietal guiding the temporal. Brain Research Reviews, 30, 66-76.

Vidyasagar, T. R. (2001). From attentional gating in macaque primary visual cortex to dyslexia in humans. Progress in Brain Research, 134, 297-312.

Vidyasagar, T. R. (2005). Attentional gating in primary visual cortex: A physiological basis for dyslexia. Perception, 34, 903-911.

Vidyasagar, T. R., \& Pammer, K. (1999). Impaired visual search in dyslexia relates to the role of the magnocellular pathway in attention. Neuroreport, 10, 1283-1287.

Witton, C., Talcott, J. B., Hansen, P. C., Richardson, A. J., Griffiths, T. D., Rees, A., et al. (1998). Sensitivity to dynamic auditory and visual stimuli predicts nonword reading ability in both dyslexia and normal readers. Current Biology, 8, 791-797.

Xu, B., Grafman, J., Gaillard, W., Ishii, K., Vega-Bermudez, F., Pietrini, P., et al. (2001). Conjoint and extended neural networks for the computation of speech codes: The neural basis of selective impairment in reading words and pseudowords. Cerebral Cortex, 11, 267-277.

Manuscript received January 5, 2005

Accepted March 5, 2006 